\title{
Teaching Network TechnOlOgies that SuPPORT INDUSTRY 4.0
}

\author{
Ishwar Singh*, Nafia Al-Mutawaly**, and Tom Wanyama*** \\ School of Engineering Technology, McMaster University \\ Hamilton, Ontario \\ isingh@mcmaster.ca* \\ almutan@mcmaster.ca** \\ wanyama@mcmaster.ca***
}

\begin{abstract}
Industry 4.0 is a combination of many elements, including distributed intelligence, network security, massive data, cloud computing, and analytics, among other things. Such elements are critical to the "Digital Factory", a term that has been recently introduced by many companies indicating a comprehensive portfolio of seamlessly integrated hardware, software and technology-based services, with the aim to enhance manufacturing productivity and improving efficiency. Typically, industrial networks enable the gathering of extensive data from production lines and plants, which are increasingly becoming distributed. The gathered data is transmitted to analysis centers where it is transformed into information and used to make better informed decisions. In addition, modern industrial networks allow plant data to be automatically filtered and transmitted to various production controllers. Ultimately, industrial networks enable Industry 4.0 to have the following benefits: improved safety, increase uptime, lower energy costs, and improved maintenance; all of which lead to manufacturing competitiveness in cyber-physical production systems supported by Smart Grid implementations. This paper presents the extent to which industrial networks are taught at the School of Engineering Technology at McMaster University. Further, the paper covers teaching methods of industrial networks and their related applications within manufacturing plants and electrical grid.
\end{abstract}

Keywords: Digital factory, experiential learning, IEC61850, Industry 4.0, industrial networks, integrated learning, smart grid

\section{INTRODUCTION}

In 2012, the government of Germany established an Industry 4.0 working group to spur a potential fourth industrial revolution, following the previous three industrial revolutions, namely: the introduction of the steam engine, electricity, and information technology. The main objective of this revolution is to develop new business models that tap the potential optimization in production and logistics caused by increased industrial automation, intelligent system monitoring, autonomous decision-making, and real-time or almost real-time communication at all levels [1]. Industrial advances independent of, but related to Industry 4.0 have blurred the boundaries between the digital and physical worlds. The terms Digital Industry and Industry 4.0 are usually used interchangeably, with the latter term adopted for this paper.

When implementing Industry 4.0, organizations should address the following factors:

- Cloud Computing: Cloud computing involves deploying groups of remote servers and software networks allowing online access to computer services and resources such as data storage, advanced data analytics, process and systems monitoring, and data access and visualization. Increased access to computing power availed to industry through cloud computing enables extensive simulations of different aspects of industrial operation, and allows processing of huge amounts of data that industry already collects but does not use adequately [3]. Taken together, this should result in better and faster decision making.

- Global Database: Industry 4.0 relies on information sharing among production and logistics centers of companies. To ensure that all the centers utilize the most current information, it is necessary to receive information from a single location, defined by Schuh et al [2] as the "single source of truth" and presented in this paper as the global company database. Since Industry 4.0 companies generate and utilize vast amounts of data, the global database is beyond what can be integrated into user devices. Therefore, it is usually implemented as part of cloud computing services.

- Automation: With the help of appropriate information processing units, automation based 
Industry 4.0 enables a bank of machines to perform tasks independently. Automation systems link the virtual information/digital environment and the physical world by integrating information processing units with sensors/actuators. To realize full Industry 4.0 benefits, companies should maximize the automation of processes.

- Networks: In order for different production centers to share information, network nodes must be physically interconnected through data communication networks. This will allow massive amounts of information (generated by industrial sensors) to be shared and sorted using various types of communications protocols. Industry 4.0 standard utilizes TCP/IP, the most common internet protocol, to link several billion devices.

It should be noted that two additional enablers related to Industry 4.0 has been largely overlooked by the literature, namely, education/training and reliable smart grid:

- Education/Training: Advances in manufacturing have to be supported by access to a highly skilled, educated workforce of technicians, trades workers and engineers. As the fourth industrial revolution takes hold, it is important that educational institutions modify their programs and develop new industrial network courses producing Industry 4.0 ready graduates.

- $\quad$ Smart Grid: The power industry is both beneficiary and enabler of Industry 4.0. From a benefits perspective, smart grid deployment permits shifting its production model from consumption-oriented generation to generation-optimized consumption, with the latter model increasing efficiency and lowering the need to invest in expensive generation capacity. On the other hand, the power industry is expected to support Industry 4.0 by supplying cost effective and reliable energy.

\section{TEACHING INDUSTRY 4.0 TECHNOLOGIES}

The School of Engineering Technology (SET) at McMaster University offers a process automation program that covers many areas of Industry 4.0. Moreover, the school delivers an energy program that covers smart grid in depth, preparing students not only to work in Industry 4.0 environment but also to support utilities to realize the benefits of Industry 4.0. This paper presents the extent to which industrial networks are taught SET. Also discussed in the paper are the methods used to teach the principles of industrial networks as well as their application in manufacturing plants, and in smart grid.

\section{MANUFACTURING PLANT INDUSTRIAL NETWORKS}

Historically, industrial networks or network protocols have been developed by different companies and later become standards. Manufacturers of industrial automation equipment continue to implement many of these protocols in devices for the following reasons [8]:

- Protocols are supported by big automation companies.

- Industrial devices that use legacy network protocols tend to have long useful life.

- Industrial network protocols exhibit a high degree of determinism with low cycle times, typically less than 15 milliseconds.

Most legacy industrial networks were designed for data communication among sensors, actuators, remote $\mathrm{I} / \mathrm{O}$ modules, and controllers (Figure 1). This data tends to be captured as short strings (small data) with many cases no more than 32 bytes per message. At the plant level of the industrial network architecture, devices share massive information, which requires high capacity networks. Therefore, most plant level networks use Ethernet based protocols such as PROFINET and Ethernet/IP (Figure 1), since Ethernet is capable of carrying large amounts of data. Also, Ethernet based protocols at the plant level make it easy to integrate the plant network and the business network, a feature required by Industry 4.0. As Ethernet is ubiquitous and cost effective, with common physical links, high speed and determinism, it is poise to becomes the de facto protocol for industrial networks.

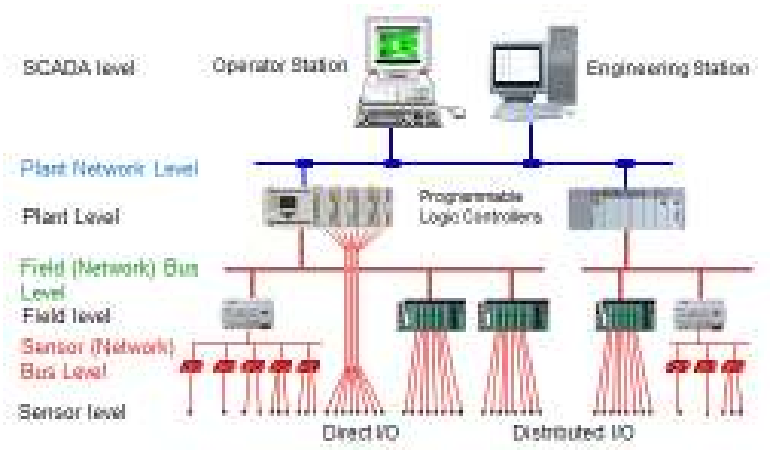

Fig. 1. Industrial network architecture

It is expected that the data flow between process level sensors/actuators and plant data centers will continuously grow. To accommodate such growth, SET has adopted Ethernet based protocols within its curriculum, as a means of integrating production facilities, supply chain and customer service centers. Various SET network courses cover Ethernet protocol topics including: Standard 
TCP/IP Ethernet, Ethernet/IP, PROFINET, High Speed Ethernet, and EtherCAT.

It is recognized that legacy networks will play an ongoing and significant role for many years to come. Accordingly, SET students are introduced to legacy networks topics which can be integrated with Ethernet based plant level networks (Figure 1). Industrial networks and controllers, a course offered by SET's process automation stream, covers many legacy networks topics including: Devicenet, CAN Bus, PROFIBUS, AS-I, Foundation Fieldbus, HART, and Modbus. Combining legacy devices with Industry 4.0 produces comprehensively skilled graduates capable of leading companies with seamless transitions from old to new technologies.

The industrial networks courses delivered by SET are based on the principle of combining lectures, laboratories and course project, a combination collectively known as "integrated learning" [7]. According to this paradigm, the theory of the networks is presented via lectures, while the network configuration and communication programming are covered through laboratories. The course project is used to integrate lectures and laboratories through an experiential learning paradigm [6].

\subsection{Industrial Networks Lectures}

Industrial networks lectures are designed to cover the basic features of the networks in reference to the Open Systems Interconnection (OSI) model. Further, lectures cover network design and implementation principles such as network cycle time, current and voltage drop estimation in network powered systems, and calculation of best location of nodes. Enquiry based learning (Socratic method), where questions are posed to students to stimulate discussions, represents a crucial element in SET's course delivery. In terms of pedagogical practice, Wanyama and Singh [6] recommended that classroom questions be designed to create an iterative backward and forward review of the course material. The authors also suggested designing questions, permitting students to relate concepts learned from different topics and to develop generalizations from the course material.

\subsection{Industrial Networks Laboratories}

Through the SET industrial networks courses, students complement lecture theory with laboratory work allowing them to better understand various network principles such as addressing structure, wiring requirements, and node power requirements. Figure 2 illustrates a typical Ethernet IP laboratory set up. The main objective of the first part of this laboratory is teach students the process of identifying useable IP addresses as well as assigning addresses to the network nodes. Other laboratories cover network configurations using software tools such as CX Configurator and RsWorx.

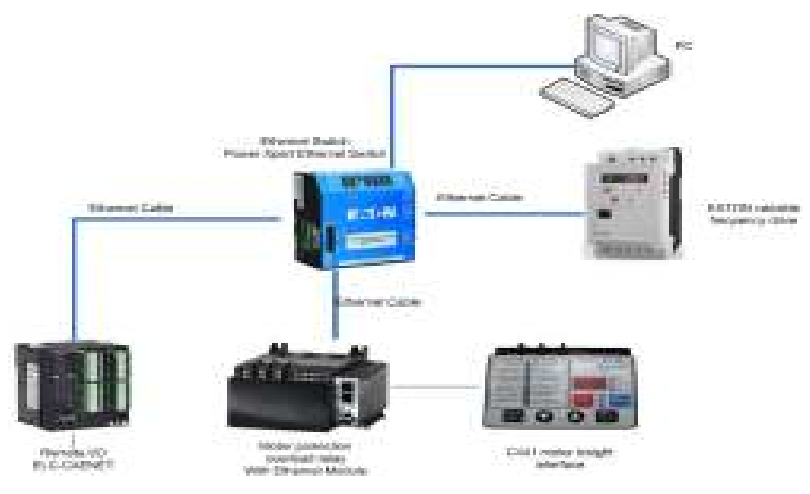

Fig. 2. Ethernet IP laboratory setup

After assigning IP addresses to the network nodes and ensuring that all nodes communicate correctly, students move on the second part of the laboratory that involves configuring the communications in the PLC programming software. In this laboratory, the software Automation Direct Productivity 3000 PLC was used. Figure 3 presents the configuration for communication between the PLC and the Eaton ELC-CAENET remote I/O module, where memory is allocated automatically to the input, output, and configuration data. Since the communication between the remote $\mathrm{I} / \mathrm{O}$ and the PLC is based on the principle of implicit messaging of Ethernet IP, there is no need for programming the communication, instead data is read and written automatically.

Implicit messaging is not supported between Automation Direct Productivity 3000 PLC and Eaton PowerXL DG1 VFD. Therefore, in this lab students have to configure a client just as they do for implicit messaging, but the reading and writing of data is implemented through explicit messages. Figure 4 shows a message instruction for reading data from the Eaton VFD.

\subsection{Industrial Networks Course Project}

The course project was designed with the objective that students utilize the knowledge acquired through lectures and laboratories, and extend their skills through experiential learning. Wanyama et al [6] suggested a project approach where students integrated Devicenet hardware with an IEC61850 device over the laboratory's Local Area Network (LAN). In another project, students integrated various Ethernet devices to establish Machine to Machine (M2M) communication. The skills learned through these projects are highly desirable in Industry 4.0 manufacturing environments. 


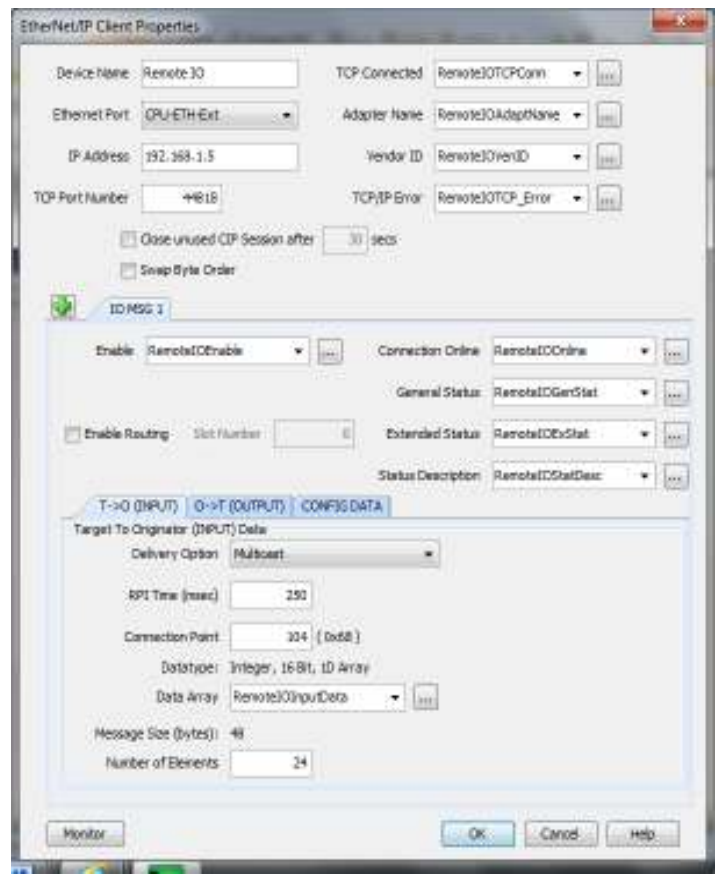

Fig. 3. Ethernet IP implicit message configuration

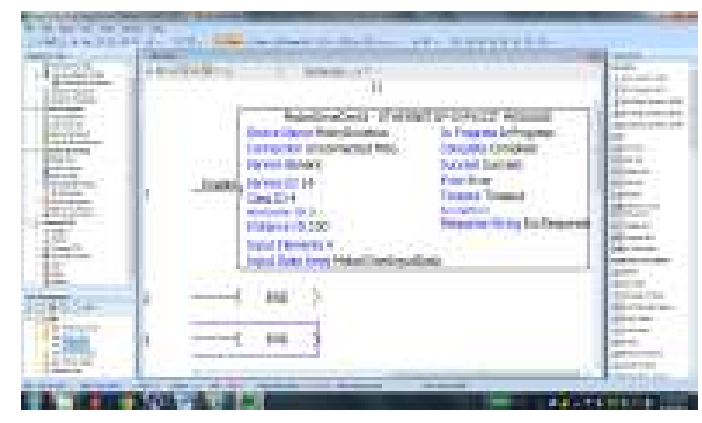

Fig. 4. Ethernet IP explicit message

\section{SMART GRID NETWORKS}

Similar to the manufacturing sector, utilities are affected by the fourth industrial revolution. Consequently, the grid has become smart leading to the need for information sharing among various energy centers. Standards such as IEC61850 have been developed to take advantage of the strength of Ethernet based protocols to streamline data sharing and information among power grid devices and energy centers.

Acknowledging the need for capable personnel to support the growth of smart grid, SET curriculum covers IEC61850 substation automation standard through various courses (Advanced System Components and Integration, Industrial Network and Protection and Control I and II). These combined lecture/lab courses were based on the general IEC61850 substation architecture (Figure 5) [4]. The Figure shows that the Merge Units (MUs) collect data from field devices, such as voltage and current transformers. Thereafter, the MUs send the data to relays, meters, and visualization points through redundant 100MB Ethernet network. Moreover, the substation architecture has an Ethernet backbone which connects the substation to other substations, control centers and remote operators [4].

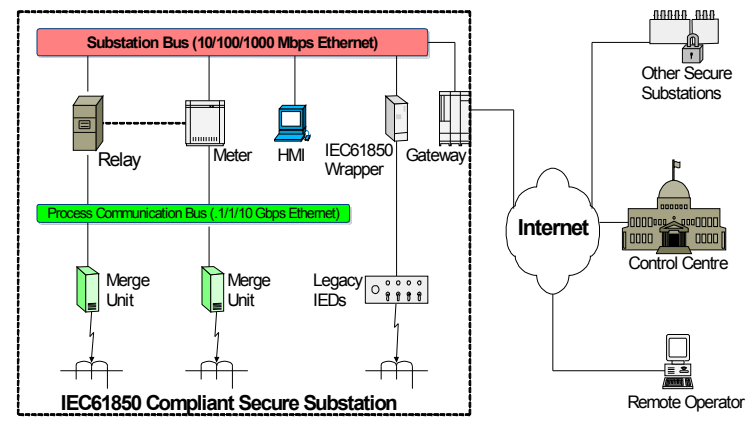

Fig. 5. Generic architecture of the IEC61850 network. (adopted from Wanyama and Singh [4])

\subsection{Advanced Power System Lab}

The Advanced Power System Lab (APSL) was developed at two sites: Mohawk College and McMaster University (located $4 \mathrm{~km}$ apart). The lab conceptual layout is shown in Figure 6. This laboratory is used by the Energy Engineering Technology program at McMaster University, and it has the following characteristics:

- Substation architecture: Each substation includes a full data aggregation centre, control room, dualredundant network (using both fiber optics and twisted pair copper), and an IP video security system. IEDs from multiple vendors and data acquisition hardware/software were installed to emulate an IEC 61850 compliant substation. Each control room (training space) is located physically next to a dedicated lab (teaching space) emulating the field side of the substation.

- Communications: Connectivity between the two substations is established via multiple media types typically used in IEC 61850 compliant substations. Connections including WiMAX, Spread Spectrum wireless, shared and dedicated data links (T1 and single-purpose fiber optics) were installed to facilitate multi-media performance testing.

- Device Network: Typical control center equipment such as the operator interface, engineering workstation, GPS time receiver, gateway and servers were installed at each substation. Protection and control devices on both substation sides includes IEDs of various types at the bay level (feeder, transformer, and generator protection) and networked I/O devices at the process level. Status 
and performance of network attached components are controllable and visible from custom, PC-based Human Machine Interfaces (HMIs) and Supervisory Control and Data Acquisition (SCADA) displays.

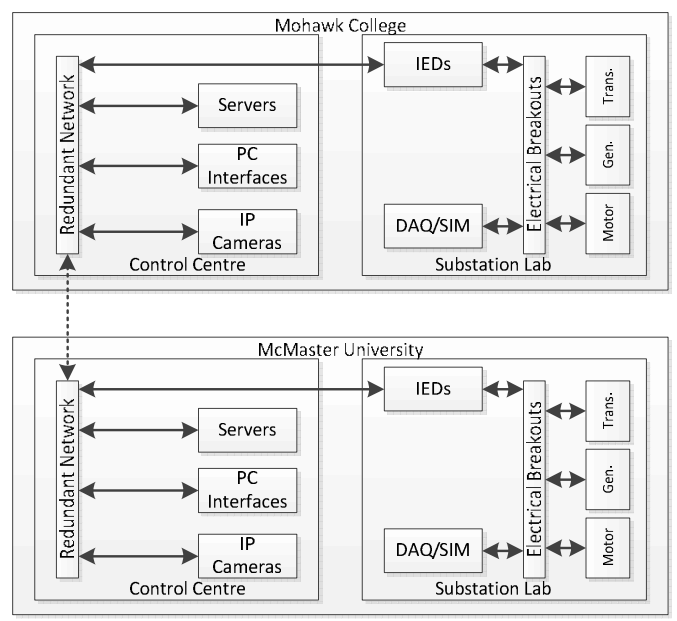

Fig. 6. APSL Conceptual layout

\subsection{Laboratories Supported by APSL}

A number of topics are delivered through the APSL, including:

- IED function verification: The APSL is used to test digital relay performance under a wide range of specified conditions to meet LDCs' system upgrade needs.

- Multi-vendor IED systems: The APSL is used to test IEDs' intercompatibility in a multi-vendor configuration and verify their collective compliance to the IEC 61850 standard [5].

- Multiple media types: The APSL's dual-site configuration is used to evaluate command cycle timing differences (between the two campuses) over a variety of media types. This includes wireless and public IP network links where singlepurpose fiber optic installations would be impractical.

- Inter-substation communication using multivendor platforms: With impacts of media types on inter-station signaling identified, the dual APSL sites was used to evaluate the performance and reliability of communication (between substations) using multiple vendors' equipment.

\section{CONCLUSION}

Industry is undergoing an information revolution, generally referred to in the literature as Industry 4.0. For companies to benefit from this revolution, they must embrace automation, cloud computing, global database that serve as a "single source of truth", combined with networking of manufacturing, logistics and customer centers. Since education/training and smart grid represent key applications of Industry 4.0 , it is vital to prepare graduates capable of assuming leading roles in supporting manufacturers and utilities.

\section{References}

[1] Benno Bunse, Henning Kagermann, and Wolfgand Wahlster, Industry 4.0: Smart Manufacturing for the Future, Germany Trade and Invest, Berlin, German, July 2014. Available as of April 12, 2015 from http://www.gtai.de/GTAI/Content/EN/Invest/_SharedDo cs/Downloads/GTAI/Brochures/Industries/industrie4.0smart-manufacturing-for-the-future-en.pdf

[2] Günther Schuh, Christina Reuter, Annika Hauptvogel , and Christian Dölle, Hypotheses for a Theory of Production in the Context of Industrie 4.0, Advances in Production Technology, Lecture Notes in Production Engineering, C. Brecher (ed.), 11 pp., 2015, ISBN 9783-319-12303-5

[3] Günther Schuh, Till Potente, Cathrin Wesch-Potente, Anja Ruth Weber", Jan-Philipp Prote, "Collaboration Mechanisms to increase Productivity in the Context of Industry 4.0", in Proc. 2nd CIRP Robust Manufacturing Conference, Katja Windt (ed.), (Bremen, Germany; 7 - 9 July 2014) , 51 pp., 2014,ISBN: 978-1-63439-362-1.

[4] Ishwar Singh and Tom Wanyama, "A Laboratory on the Configuration of Electric Power Substation three Phase Power Supply", in Proc. $3^{\text {rd }}$ Interdisciplinary Engineering Design Education Conference (IEDEC), (Santa Clara, CA; 4-5 March 2013), 153 pp., 2013. ISBN: 978-1-4673-5113-3.

[5] R. Aguilar and J. Ariza, "Integration and testing challenges of IEC 61850 multivendor protection schemes," in Developments in Power System Protection, 2010 .

[6] Tom Wanyama and Ishwar Signh, "Laboratory Based Project for Experiential Learning in PLC Systems Integration and PLC Systems Data Access", in Proc. CEEA Canadian Engineering Education Conf., CEEA2014, (Canmore, Alberta; 8-11 June 2014), 2014.

[7] Tom Wanyama, and Ishwar Singh, "Integrating Lectures, Laboratories and Course Projects", In Proc. of the International Conference on Engineering Education and Research, ICEER2014, Mohamed Bakr and Ahmed Elsharabasy (ed) (Hamilton, Ontario; Canada, 25-26 August 2014), 34 pp., 2014

[8] Zhihong Lin, Stephanie Pearson, "An inside look at industrial Ethernet communication protocols", Texas Instruments, Dallas, Texas, November 2013, Available as of April 12, 2015 from http://www.ti.com/lit/wp/spry254/spry254.pdf 